\title{
Otimização de rotas em redes MIMO múltiplos saltos com métricas inter-camadas
}

\author{
Ricardo Borges, Anelise Munaretto, Mauro Fonseca e Richard Demo Souza
}

\begin{abstract}
Resumo-Este artigo investiga o uso de técnicas MIMO na escolha de rotas de comunicação em uma rede sem fio múltiplos saltos. Considerou-se que o enlace entre dois nós pode ser estabelecido usando um dos dois diferentes esquemas MIMO, STBC ou V-BLAST, e uma das várias ordens de modulação propostas. Simulações computacionais foram realizadas a fim de identificar a melhor configuração para diferentes condições de canal (valores de SNR) para cada enlace existente entre dois nós. Assim, qualquer rota entre uma origem e um destino, formada por múltiplos saltos, é otimizada a fim de maximizar a taxa de transferência de dados. Os resultados das simulações indicam que a escolha da melhor rota entre dois pontos considerando a seleção adaptativa da técnica MIMO e da ordem de modulação, em cada enlace, é capaz de aumentar consideravelmente a taxa de transferência de dados em uma rede múltiplos saltos.
\end{abstract}

Palavras-Chave-MIMO, Inter-camadas, Redes sem fio múltiplos saltos.

Abstract-This paper investigates the use of MIMO techniques to select routes in a wireless multihop network. We consider that the link between any two nodes can be established using one of two different MIMO schemes, STBC or V-BLAST, and one of many modulation orders. Computer simulations were carried out in order to identify the best configuration for different channel conditions (SNR values) for each link between each pair of nodes. Then, any source-to-destination route, which may be composed of multiple hops, is optimized in order to maximize the data throughput. Simulation results indicate that the adaptive selection of the MIMO technique and the modulation order for each link can increase considerably the data throughput in a multihop network.

Keywords-MIMO, Cross-layer, Multihop wireless network.

\section{INTRODUÇÃO}

Redes com tecnologia sem fio têm atraído muita atenção da comunidade científica principalmente das áreas de comunicações e computação. Na verdade, a natureza dinâmica das redes sem fio impõe grandes desafios, como limitação do alcance e variação dos enlaces sem fio. Novas tecnologias sem fio têm surgido como soluções alternativas para alguns destes desafios. Duas das mais avançadas são as técnicas de múltiplas antenas, também conhecida como técnicas MIMO, e o suporte a redes sem fio múltiplos saltos. Uma rede sem fio múltiplos saltos é capaz de se auto-organizar e se autoconfigurar dinamicamente, onde os nós estabelecem e mantém a conectividade entre eles automaticamente, aumentando a cobertura e a confiabilidade da rede. Contudo, para atingir

Ricardo Borges, Anelise Munaretto e Richard Demo Souza, CPGEI, UTFPR, Curitiba, Brasil 80230-901, Email: r7borges@gmail.com; anelise@cpgei.cefetpr.br; richard@cpgei.cefetpr.br. Mauro Fonseca PPGIA, PUCPR, Curitiba, Brasil 80215-901, Email: mauro.fonseca@ ppgia.pucpr.br. Este trabalho foi parcialmente financiado pela Fundação Araucária (01/20069638) e pelo CNPq (472977/2007-5) o melhor desempenho, é fundamental que estas tecnologias operem de acordo com as condições de propagação do meio, condições estas que podem estar em constante variação.

O conceito de inter-camada (cross-layer) é baseado em uma arquitetura onde diferentes camadas de comunicação podem trocar informações a fim de melhorar o desempenho global da rede [1]. Resultados promissores obtidos utilizando arquiteturas inter-camadas, iniciaram inúmeras pesquisas nesta área. Akyildiz e Wang [2] investigaram esquemas de otimização utilizando protocolos entre diferentes camadas, com objetivo de esclarecer problemas ainda em aberto e oferecer novas abordagens. Em [3], é apresentado que uma otimização dos recursos em uma rede sem fio pode ser alcançada através de plataformas que integrem informação de múltiplas camadas.

Além disso, os autores em [4] propuseram uma abordagem inter-camada que utiliza medidas de interferência e taxa de sucesso de pacote (PSR - Packet Success Rate) como percebido pela camada física, e a taxa de dados como uma métrica de roteamento. Assim, a camada de roteamento também otimiza a potência usada para transmitir pacotes, dependendo do próximo destino, de maneira a maximizar a capacidade da taxa de transferência de dados global. Em [6], quatro modelos de canais para comunicações sem fio múltiplos saltos são propostos. As simulações mostram que o uso de canais múltiplos saltos podem melhorar significativamente o desempenho, porém estas melhorias são muito sensíveis para a localização de terminais intermediários abaixo da potência total. Todos os quatro canais de múltiplos saltos superam o canal de referência de salto simples, desde que as formas de transmissão dos terminais intermediários (relays) sejam escolhidos de maneira eficaz, com canais múltiplos saltos contendo diversidade, superando os canais múltiplos saltos sem diversidade.

Neste artigo é proposta uma otimização de rotas em redes MIMO múltiplos saltos com métricas inter-camadas, com a meta de maximizar a taxa de transferência de dados da rede. Assume-se que cada nó pertencente à rede é capaz de se comunicar utilizando diferentes ordens de modulações e técnicas de transmissão MIMO. Além disso, os nós são capazes de mudar o esquema de transmissão de acordo com as condições do enlace. Esse esquema de transmissão adaptativa, ou modulação adaptativa, poderia por exemplo, ser implementado por meio de tecnologia de rádio definido por software. Assim, baseado no conhecimento do estado do canal de cada enlace, que pode ser utilizado dentro da rede múltiplos saltos, um algoritmo de roteamento pode selecionar a melhor rota e o melhor esquema de transmissão a ser utilizado em cada salto, a fim de maximizar a taxa de transferência de dados entre a origem 
e o destino.

Simulações computacionais mostram que um considerável aumento da taxa de transferência de dados, pode ser obtido através da proposta de otimização inter-camada, incluindo parâmetros das camadas física, de enlace e de rede. Além disso, verifica-se que, utilizando seleção adaptativa da técnica de transmissão MIMO, é possível obter ganhos consideráveis em ambientes onde cada enlace experimenta condições de canal diferentes. Este tipo de ambiente facilmente encontrado, devido à natureza imprevisível e inconstante do canal sem fio.

O restante deste artigo é organizado da seguinte forma. A Seção II apresenta uma breve discussão das características das redes sem fio múltiplos saltos. A Seção III descreve alguns detalhes das técnicas MIMO utilizadas neste artigo. A Seção IV apresenta e discute os resultados das simulações utilizando diferentes modulações e técnicas MIMO para as topologias ponto a ponto e múltiplos saltos. Finalmente, a Seção V contém os comentários finais.

\section{REDES MÚLTIPLOS SALTOS}

A comunicação direta entre dois dispositivos é dita ser uma conexão ponto a ponto, onde o primeiro dispositivo é a origem e o outro é o destino. Quando dados são enviados através de dispositivos intermediários (relays) entre a origem e o destino, esta é denominada rede múltiplos saltos. Em redes sem fio múltiplos saltos, desde que os enlaces intermediários estejam mais próximos uns dos outros do que o enlace direto entre a origem e o destino, a atenuação é consideravelmente reduzida (uma vez que é função de uma potência da distância entre os enlaces). Portanto, usando múltiplos caminhos é possível aumentar a cobertura da rede, alcançando usuários que não estariam disponíveis numa conexão ponto a ponto. Outra vantagem é a possibilidade de diminuir a potência de transmissão e a interferência [7], [8].

Uma das topologias de redes sem fio múltiplos saltos é conhecida como redes ad hoc. Uma rede ad hoc é uma rede múltiplos saltos dinâmica e auto-organizável [9]. Os nós sem fio devem ser capazes de se adaptar à topologia dinâmica e às condições variáveis da rede. Além de ser uma rede múltiplos saltos, uma rede ad hoc pode ser móvel e utilizada em lugares onde redes fixas poderiam não estar disponíveis. Uma das dificuldades é determinar e manter controle das rotas nesta topologia de rede altamente dinâmica. Uma nova topologia que vem sendo altamente desenvolvida é a usada em redes em malha (mesh). As redes em malha apresentam uma hierarquia na sua arquitetura, através da implementação de alguns nós dedicados, conhecidos como roteadores mesh [10]. Uma rede em malha, embora siga uma hierarquia, pode ser dinâmica, auto-organizável e auto-configurável, com nós estabelecendo automaticamente uma rede ad hoc enquanto mantém a conexão na topologia em malha.

Redes em malha são projetadas considerando nós clientes e nós roteadores, interconectados por enlaces sem fios, formando uma rede múltiplos saltos. Os roteadores mesh tem mínima mobilidade e formam um backbone sem fio [10]. O tráfego é organizado nos clientes, passa pelo backbone sem fio, que oferece conexões múltiplos saltos para outros clientes, e conexões para gateways com acesso à internet ou em redes externas (formando backhaul). Além disso, os clientes mesh podem também operar como roteadores. Porém, o hardware e o software deles são mais simples e limitados em relação àqueles utilizados pelos roteadores [11].

Em uma rede múltiplos saltos, todo caminho que passa por um ou mais saltos entre a origem e o destino deve ser conhecido. Um protocolo de roteamento pode ser utilizado com o objetivo de descobrir a melhor rota para cada destino da rede. Incialmente, um protocolo de roteamento executado em um nó realiza a descoberta de seus nós vizinhos e então da topologia da rede. Protocolos de roteamento usualmente caracterizam a rede com uma métrica simples, como a distância em número de saltos ou o atraso, e utilizam o algoritmo de escolha do menor caminho para a descoberta da rota. Dependendo da métrica utilizada ou da combinação de várias delas, pode ser realizada a otimização dos recursos da rede. Uma métrica é um padrão de medida utilizado por algoritmos de roteamento para determinar o caminho ótimo até um destino. Os algoritmos de Bellman-Ford[12] e Dijkstra[13] são utilizados por protocolos de roteamento a fim de obter a melhor rota possível, através do cálculo do caminho de menor custo. A diferença entre estes dois algoritmos é o fato que o algoritmo de Dijkstra considera um grafo com arestas cujos pesos são não negativos. Este algoritmo, em sua forma original, resolve o problema do menor caminho em um grafo diretivo, cujas arestas têm um dado peso. Basicamente, pode-se ter uma métrica aditiva, uma métrica multiplicativa e uma métrica côncava [14]. Muitos protocolos de roteamente foram recentemente propostos para redes ad hoc [15] e em malha sem fio[16]. Porém, especificação de um protocolo de roteamento está fora do escopo deste trabalho.

Neste artigo, considera-se uma rede em malha sem fio com nós estacionários (roteadores mesh), sendo que cada nó já conhece a topologia da rede. Além disso, faz-se uso do algoritmo de Dijkstra com uma métrica aditiva. Uma métrica aditiva é uma métrica que agrega as medidas ao longo do percurso, ou seja, soma os valores pertencentes a todas as arestas do percurso. Na proposta deste artigo, a métrica é uma função custo derivada de uma expressão que calcula a taxa de transferência de dados dos enlaces, selecionando rotas capazes de maximizar tais taxas. Um método efetivo é utilizar a taxa de erro de quadros (FER - Frame Error Rate) entre os enlaces. Sendo assim, o estado do canal (em que se considera o valor de SNR) entre todos os pares de nós é atualizado periodicamente e os valores de FER são estimados para cada estado do canal [17].

\section{TÉCNICAS MIMO}

Em comunicações sem fio, os desafios, devido aos fortes efeitos do desvanecimento induzido pelo canal sem fio, são muitos[18], [19]. Uma das maneiras de combater o desvanecimento é inserindo diversidade no sistema de comunicação. Pode-se obter diversidade de várias formas, como por exemplo, na freqüência, no tempo ou no espaço. No caso de diversidade espacial, é possível utilizar múltiplas antenas na transmissão, múltiplas antenas na recepção, ou os dois casos 
juntos. Este último método caracteriza um sistema MIMO (Multiple Input Multiple Output). Dois aspectos importantes de sistemas MIMO podem ser citados [18]:

- A utilização de múltiplas antenas pode levar ao aumento da capacidade ou da eficiência espectral;

- Aumentando a capacidade do canal, por meio de técnicas MIMO, implica em um aumento da complexidade computacional, mantendo fixo outros recursos de comunicação primários, como a largura de banda e a potência utilizadas.

Embora existam outros métodos interessantes, este artigo considera apenas os dois métodos mais utilizados e conhecidos, que são as técnicas MIMO STBCs (Space-Time Block Codes) e BLAST (Bell Laboratories Layered Space-Time).

\section{A. $S T B C$}

O STBC foi primeiramente proposto por Alamouti em [20]. A idéia é aplicar redundância espacial através de antenas transmissoras, com o objetivo de aumentar a proteção contra o desvanecimento e minimizar a probabilidade de outage [18]. Portanto, a meta é diminuir a probabilidade de erro.

A transmissão é realizada em blocos, onde o código espaçotemporal é definido por uma matriz de transmissão. Considere $l$ como sendo o número de símbolos transmitidos por bloco e $m$ o número de time slots por bloco envolvidos na transmissão. Um STBC, utilizando $N_{t}$ antenas transmissoras, oferece uma taxa de código definida por $l / m$. Além disso, para uma transmissão eficiente, os símbolos são representados na forma complexa. Em seguida, para possibilitar a utilização de processamento linear no receptor, a matriz de transmissão deve satisfazer a condição de ortogonalidade complexa. Há duas estratégias de projetos diferentes [18]. A primeira é o projeto ortogonal, onde a matriz de transmissão é quadrada e $N_{t}=2$, e que satisfaz a condição de complexidade ortogonal tanto no espaço quanto no tempo [20]. O código de Alamouti é um exemplo de um STBC ortogonal. O outro é o projeto ortogonal generalizado, cuja matriz não é quadrada, satisfazendo apenas a condição de complexidade ortogonal temporal. Assim, $N_{t}$ pode ser maior que 2 e a taxa de código é menor que 1 [21].

No restante deste artigo, é considerado apenas o STBC de Alamouti, que obtem diversidade completa com taxa de código unitária e, atualmente, é o código espaço-temporal mais popular. No STBC de Alamouti os símbolos são processados em pares. A cada dois intervalos de tempo, o codificador libera dois diferentes símbolos para serem transmitidos por cada uma das duas antenas. $\mathrm{O}$ receptor também processa os símbolos em pares. O decodificador usa o conhecimento do canal entre cada antena transmissora e receptora, para, através de processamento linear, estimar os símbolos mais prováveis que alimentaram o codificador [20]. A fim de simplificar a notação, a partir deste ponto, é utilizado o termo STBC para referir-se ao esquema STBC de Alamouti.

\section{B. BLAST}

A arquitetura BLAST emprega múltiplas antenas transmissoras e múltiplas antenas receptoras, com uma diferença fundamental em relação aos STBCs. Ao utilizar-se o BLAST, o objetivo é aumentar a eficiência espectral pelo aumento da taxa de transmissão. $N_{t}$ símbolos são simultaneamente transmitidos a cada instante de símbolo. A taxa de transmissão pode aumentar linearmente com o número de antenas transmissoras quando utilizado BLAST [22]. Diferentes implementações de BLAST podem ser encontrados na literatura, porém neste artigo, o foco está no V-BLAST, o qual foi utilizado na primeira implementação prática de um sistema de comunicação sem fio MIMO [23].

$\mathrm{Na}$ arquitetura V-BLAST[24], uma simples seqüência de dados é dividida em $N_{t}$ subseqüências, usando um conversor serial-paralelo, onde cada subseqüência é separadamente codificada e modulada. As subsequiências são então transmitidas simultaneamente pelas $N_{t}$ antenas transmissoras. Cada antena receptora recebe todos os símbolos transmitidos por cada uma das $N_{t}$ antenas transmissoras. Após, os sinais são processados para a detecção de cada subseqüência transmitida. É importante observar que o receptor deve utilizar $N_{r} \geq N_{t}$ antenas receptoras para ser capaz de separar e detectar os símbolos transmitidos.

O detector V-BLAST ótimo é o detector de máxima verossimilhança, cuja complexidade computacional aumenta exponencialmente com o número de antenas transmissoras. Na prática, dois detectores de menor complexidade podem ser empregados no receptor: Forçagem a zero (ZF - Zero-Forcing) e Minimum Mean Square Error (MMSE). A implementação do algoritmo ZF é mais simples do que a implementação do MMSE, porém apresenta desempenho inferior. A próxima seção utiliza-se do detector ZF, onde um símbolo é primeiramente detectado e, em seguida, subtraído dos sinais recebidos (cancelamento sucessivo) [23]. Este procedimento é repetido até que todos os símbolos transmitidos sejam detectados.

\section{ESQUEMA DE TRANSMISSÃO MIMO ADAPTATIVA}

Nesta seção propõe-se um esquema de transmissão MIMO adaptativa, o qual é capaz de selecionar a melhor ordem de modulação e o melhor esquema MIMO, para uma dada relação sinal ruído (SNR). Para tal, consideram-se quatro diferentes modulações (BPSK, QPSK, 8PSK e 16QAM) e duas técnicas MIMO (STBC e V-BLAST). O esquema MIMO utiliza-se de duas antenas transmissoras $\left(N_{t}=2\right)$ e quatro antenas receptoras $\left(N_{r}=4\right)$. O canal entre as antenas transmissoras e receptoras é considerado como do tipo Rayleigh quaseestático, com ganhos $h_{i j}$ entre a antena transmissora $i$ e a antena receptora $j$, modelados por uma variável aleatória Gaussiana complexa, com média zero e variância unitária. Os receptores estão sob ruído como um processo aditivo, branco e gaussiano (AWGN) com variância $\sigma_{n}^{2}$. Além disso, o quadro de transmissão é formado por 256 símbolos.

A eficiência espectral $\nu$ das técnicas MIMO consideradas podem ser escritas como:

$$
\nu=\log _{2} M \times S,
$$

onde $M$ é o número de símbolos na constelação da modulação em uso, $S=N_{t}$ para o V-BLAST e $S=1$ para STBC, 


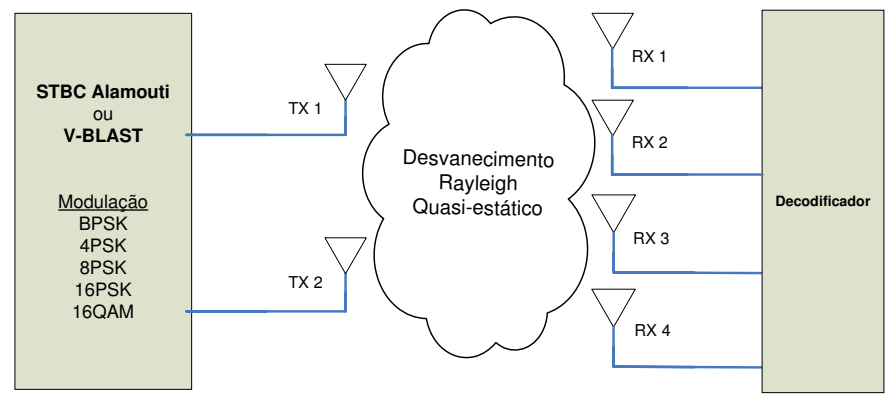

Fig. 1. TX, RX e Canal

supondo-se também largura de banda unitária. Portanto, considerando um protocolo stop-and-wait simples [25] e descartando qualquer outro atraso, a taxa de transferência de dados, pelos métodos de transmissão utilizados aqui, pode ser dada por:

$$
\Gamma=\nu(1-\mathrm{FER}),
$$

onde FER é a taxa de erro de quadro, considerando uma dada modulação e técnica MIMO.

Simulações computacionais foram realizadas para determinar o desempenho em termos de $\Gamma$ versus $S N R$ para todas as combinações de ordens de modulação e técnicas MIMO consideradas nesta seção (totalizando oito diferentes combinações). Baseado nestes resultados, a Tabela I apresenta a seleção das melhores configurações de técnicas MIMO e modulação para diferentes valores de SNR. Nesta tabela, mostram-se também os menores e maiores valores de $\Gamma$ obtidos por esta seleção da melhor configuração dentro de um dado intervalo de SNR.

TABELA I

SELEÇÃO DAS MELHORES ORDENS DE MODULAÇÃO E TÉCNICAS MIMO PARA UM DADO INTERVALO DE SNR

\begin{tabular}{ccccc}
\hline \hline $\begin{array}{c}\text { MIMO } \\
\text { Esquema }\end{array}$ & $\begin{array}{c}\text { SNR } \\
(\mathrm{dB})\end{array}$ & $\begin{array}{c}\text { Modulação } \\
\text { Tipo }\end{array}$ & $\begin{array}{c}\text { Menor } \Gamma \\
\text { (bits/s/Hz) }\end{array}$ & $\begin{array}{c}\text { Maior } \Gamma \\
(\mathrm{bits} / \mathrm{s} / \mathrm{Hz})\end{array}$ \\
\hline STBC & $1-4$ & BPSK & 0.46 & 0.95 \\
STBC & $5-10$ & 4PSK & 1.03 & 2.00 \\
STBC & 11 & 8PSK & 2.08 & 2.08 \\
V-BLAST & $12-14$ & 4PSK & 2.73 & 3.56 \\
STBC & $15-17$ & 16QAM & 3.80 & 3.98 \\
V-BLAST & $18-19$ & 8PSK & 4.60 & 5.21 \\
V-BLAST & $\geq 20$ & 16QAM & 5.93 & 8.00 \\
\hline
\end{tabular}

Analisando a Tabela I pode-se verificar que para regiões com baixos valores de SNR o esquema baseado no STBC apresenta melhor desempenho que o esquema baseado no VBLAST, este que, por sua vez, supera o esquema baseado no STBC para altos valores de SNR. Contudo, para os valores de SNR entre 12 e $14 \mathrm{~dB}$, valores considerados relativamente baixos nesta comparação, nota-se que o V-BLAST utilizando modulação 4-PSK tem melhor desempenho que o STBC com qualquer modulação. A Figura 2 mostra a taxa de transferência de dados obtida pela proposta de um esquema com transmissão adaptativa, para diferentes valores de SNR. As diferentes marcas na curva representam as diferentes estratégias de transmissão a serem utilizadas para obter melhor taxa de dados.

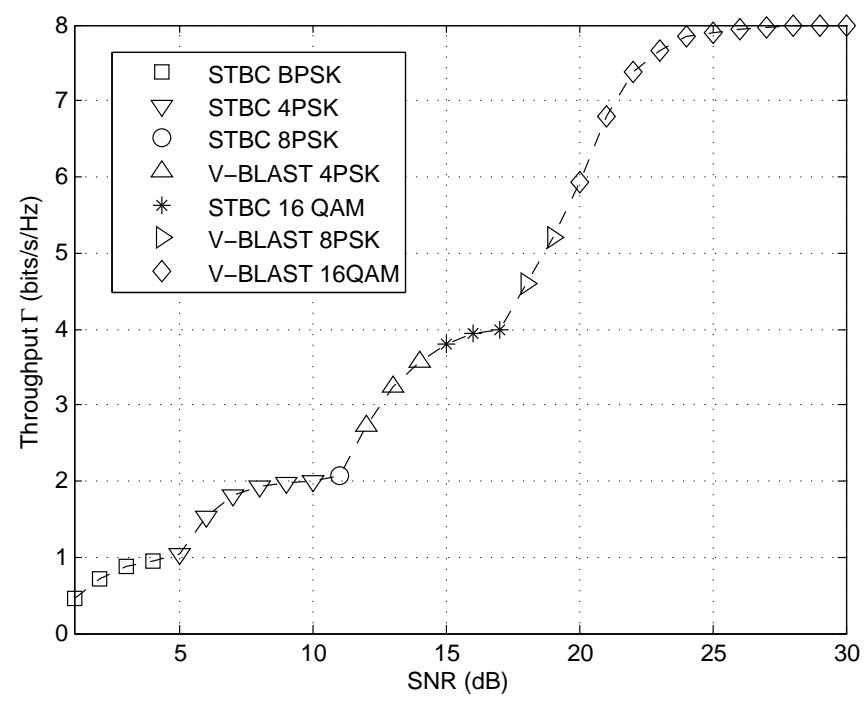

Fig. 2. Throughput $\Gamma$ versus SNR para o esquema de transmissão adaptativa definido na tabela I. As diferentes marcas na curva representam as diferentes estratégias de transmissão a serem usadas.

Além disso, é importante salientar que, considerando todos os valores de SNR e os mesmos parâmetros de transmissão, um sistema que usa uma técnica MIMO fixa ou uma única ordem de modulação, nunca atingirá uma taxa de transmissão tão boa a de um esquema adaptativo como o definido na Tabela I.

\section{TRANSMISSÃO ADAPTATIVA EM REDES SEM FIO MÚLTIPLOS SALTOS}

Suponha que o esquema de transmissão adaptativa, caracterizado pela Tabela I, é utilizado por rádios (tratados aqui como nós) pertencentes a uma rede sem fio múltiplos saltos. Investiga-se o efeito no desempenho da rede quando o esquema de transmissão apropriado é selecionado em cada salto. Para isso, assume-se que o nó origem conhece os valores de SNR de todos os enlaces da rede. Baseado nesta informação, e nos dados apresentados na Tabela I, a origem deve selecionar a rota capaz de maximizar a taxa de transferência de dados. Para o cálculo da melhor rota, utiliza-se o algoritmo de Dijkstra, com métrica aditiva. Além disso, assume-se que os nós não são capazes de transmitir e receber dados ao mesmo tempo.

A métrica utilizada para selecionar a melhor rota foi o atraso. $\mathrm{O}$ atraso foi calculado como o inverso da taxa de transferência de dados estimada. Ao considerar apenas uma transmissão por vez para cada nó, também se simplificou a camada de enlace.

Nota-se que, baseado nos resultados apresentados na Tabela I, em casos onde todos os enlaces apresentam condições ruins de canal (baixos valores de SNR), provavelmente todos os enlaces utilizariam STBC. Já separando todos os enlaces com boas condições de canal (altos valores de SNR), certamente todos usariam V-BLAST. Estes dois casos em separado não apresentariam análises e resultados muito interessantes, pois eles apenas evidenciariam as vantagens da utilização da modulação adaptativa, a qual já é bem conhecida. Entretanto, num cenário mais realístico, diferentes enlaces apresentarão significativas diferenças quanto às condições de canal para cada enlace. 


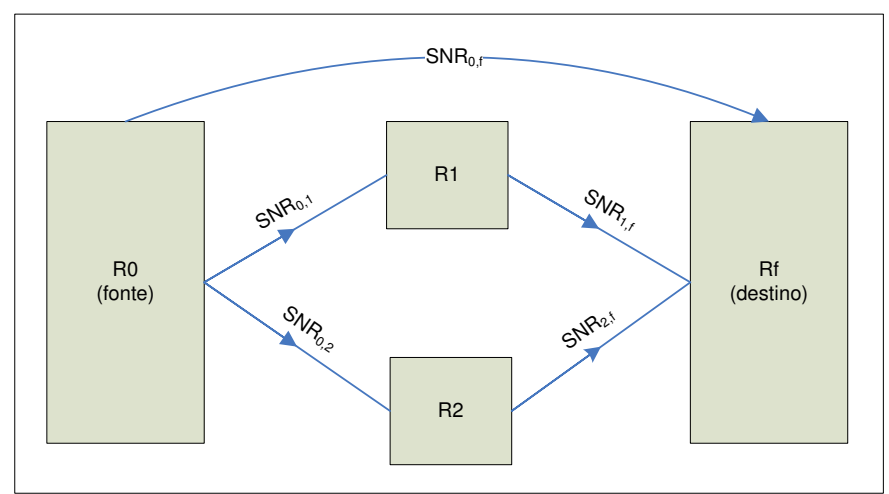

Fig. 3. Rede múltiplos saltos com 4 nós.

TABELA II

VALORES DE SNR PARA OS DIFERENTES ENLACES DA FIGURA 3, COM SUAS MELHORES ESTRATÉGIAS DE TRANSMISSÃO CORRESPONDENTES, throughput E ATRASO.

\begin{tabular}{ccccc}
\hline \hline Enlace & SNR(dB) & Melhor estratégia & $\Gamma(\mathrm{bps})$ & Atraso (s/bits) \\
\hline R0-R1 & 26 & V-BLAST 16QAM & 7.95 & 0.126 \\
R0-R2 & 10 & STBC 4PSK & 2.00 & 0.500 \\
R0-Rf & 1 & STBC BPSK & 0.46 & 2.174 \\
R1-R2 & 19 & V-BLAST 8PSK & 5.21 & 0.192 \\
R1-Rf & 2 & STBC BPSK & 0.72 & 1.389 \\
R2-R1 & 19 & V-BLAST 8PSK & 5.21 & 0.192 \\
R2-Rf & 5 & STBC 4PSK & 1.03 & 0.971 \\
\hline
\end{tabular}

Portanto, em tais redes alguns enlaces terão baixos valores de SNR, enquanto outros enlaces terão altos valores. $\mathrm{O}$ foco deste trabalho é neste tipo de cenário.

Considere agora uma rede com quatro nós, um sendo a origem, dois nós intermediários e um destino, como mostra a Figura 3. Os diferentes enlaces são caracterizados por diferentes valores de SNR, conforme Tabela II. Também estão presentes nesta tabela a melhor estratégia de transmissão para cada enlace, de acordo com os resultados da Seção IV, e a taxa de transferência de dados.

Nota-se que, nestes cenários cada nó calcula a rota para descobrir o caminho para o nó destino. Para isso um nó realiza uma descoberta da topologia e calcula as medidas da métrica. A seguir, baseado nos dados apresentados na Tabela II, o algoritmo de Dijkstra calcula e fornece a seguinte seqüência de saltos, capaz de maximizar a taxa de dados e minimizar o atraso: R0-R1, R1-R2, R2-Rf. É possível perceber que nesta rota três diferentes modulações foram utilizadas (16QAM, 8PSK e 4PSK), além dos dois esquemas MIMO (V-BLAST e STBC). O atraso total neste caso é de $1.289 \mathrm{~s} / \mathrm{bit}$. Se fosse um sistema apenas com modulação adaptativa e com esquema MIMO fixo, o atraso total seria de $1.470 \mathrm{~s} / \mathrm{bit}$ para o STBC e $2.85 \mathrm{~s} / \mathrm{bit}$ para o V-BLAST. Assim, pode-se confirmar que a utilização de esquemas MIMO adaptativos traz consideráveis ganhos. Além disso, se múltiplos saltos não fossem permitidos, ou seja, a origem se comunicando diretamente com o nó destino, este atraso seria de $2.174 \mathrm{~s} /$ bit, o que também mostra as vantagens de uma rede múltiplos saltos.

A Figura 4 mostra um cenário diferente, com seis nós, sendo caracterizada na Tabela III. Aplicando o algoritmo de Dijkstra, como no exemplo anterior, a seqüência de saltos que
TABELA III

VALORES DE SNR PARA OS DIFERENTES ENLACES DA FIGURA 4, COM SUAS MELHORES ESTRATÉGIAS DE TRANSMISS ÃO CORRESPONDENTES, throughput E ATRASO.

\begin{tabular}{ccccc}
\hline \hline Enlace & SNR(dB) & Melhor estratégia & $\Gamma(\mathrm{bps})$ & Atraso (s/bits) \\
\hline R0-R1 & 30 & V-BLAST 16QAM & 8.00 & 0.125 \\
R0-R2 & 24 & V-BLAST 16QAM & 7.80 & 0.128 \\
R0-R3 & 5 & STBC 4PSK & 1.03 & 0.971 \\
R0-R4 & 5 & STBC 4PSK & 1.03 & 0.971 \\
R0-Rf & 1 & STBC BPSK & 0.46 & 2.174 \\
R1-R2 & 19 & V-BLAST 8PSK & 5.21 & 0.192 \\
R1-R3 & 11 & STBC 8PSK & 2.10 & 0.476 \\
R1-R4 & 5 & STBC 4PSK & 1.03 & 0.971 \\
R2-R3 & 15 & STBC 16QAM & 3.80 & 0.260 \\
R2-R4 & 6 & STBC 4PSK & 1.50 & 0.700 \\
R3-R4 & 14 & V-BLAST 4PSK & 3.50 & 0.285 \\
R3-Rf & 5 & STBC 4PSK & 1.03 & 0.971 \\
R4-Rf & 10 & STBC 4PSK & 2.00 & 0.500 \\
\hline
\end{tabular}

maximiza a taxa de dados e minimiza o atraso é: R0-R2, R2R3, R3-R4, R4-Rf. Utilizando a rota otimizada, o atraso total é $1.173 \mathrm{~s} /$ bits. No caso da não utilização do esquema MIMO adaptativo, apenas da modulação adaptativa, então o melhor resultado seria $1.242 \mathrm{~s} /$ bit para o V-BLAST e $1.309 \mathrm{~s} / \mathrm{bit}$ para o STBC. Utilizando um simples salto entre a origem e o destino, este atraso seria de $2.174 \mathrm{~s} /$ bit. Novamente, fica claro que, com a utilização de múltiplos saltos, complementado pela adaptação do sistema de transmissão (as técnicas MIMO e ordens de modulação), aumentam-se os ganhos em relação a um sistema com apenas um simples salto disponível. Além disso, é interessante notar que neste último exemplo quatro diferentes estratégias de transmissão foram utilizadas para enviar os dados do nó R0 até o nó Rf, uma para cada salto.

\section{Conclusões}

Neste artigo investigou-se o uso de esquemas de modulação e técnicas MIMO adaptativas em uma rede múltiplos saltos.

Primeiramente, uma comunicação MIMO ponto a ponto foi avaliada. O objetivo foi obter a taxa de erro de quadro (FER) para as duas diferentes técnicas MIMO (Alamouti e V-BLAST), utilizando duas antenas transmissoras e quatro antenas receptoras, para quatro diferentes modulações (BPSK, 4PSK, 8PSK e 16QAM). Os resultados mostraram as melhores configurações - entre as duas técnicas MIMO e as modulações consideradas - para cada valor da relação sinal ruído (SNR) presente no enlace entre o transmissor e o receptor. Baseado nestes resultados, cada nó poderia selecionar a melhor configuração, otimizando assim os recursos de rede. Ou seja, cada nó seria capaz de adequar seu modo de transmissão de acordo com as variações do canal.

A avaliação do desempenho apresentou os ganhos obtidos utilizando múltiplos saltos com um sistema de transmissão adaptativa, comparado com um esquema sem nenhum sistema adaptativo e ponto a ponto.

Nos trabalhos seguintes, será dada continuação a investigação dos benefícios e vantagens do roteamento utilizando métricas inter-camadas, considerando maior número de cenários. O próximo passo será o projeto de um protocolo de roteamento, avaliando desafios importantes 


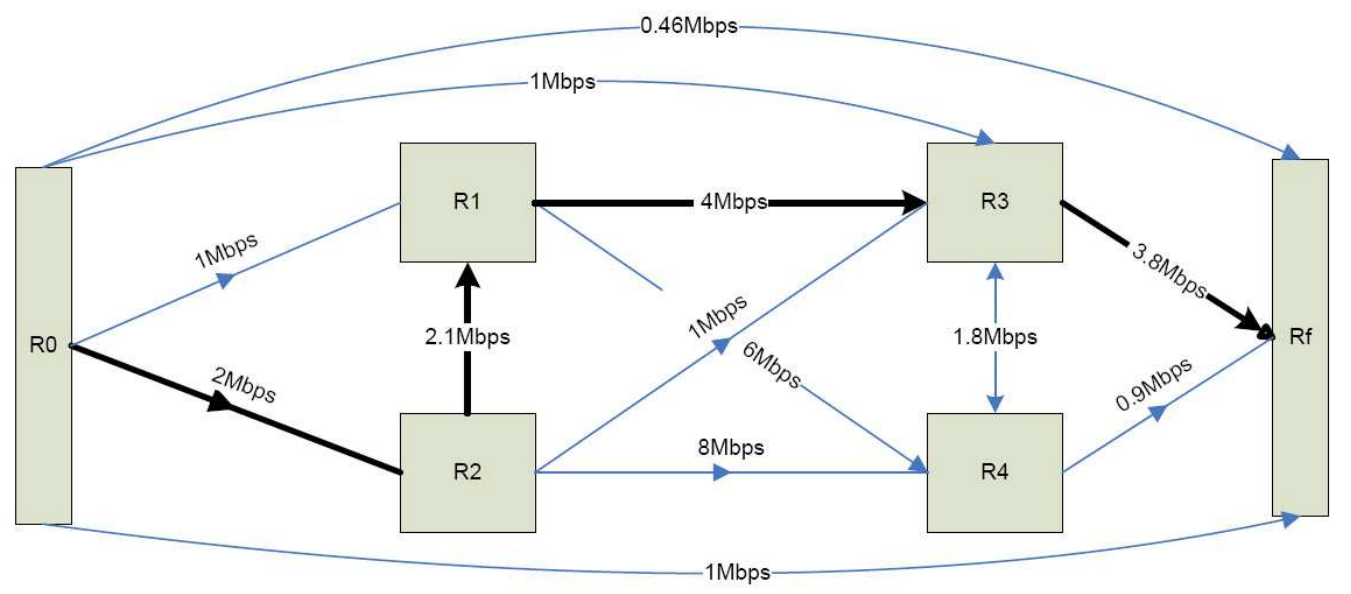

Fig. 4. Rede múltiplos saltos com 6 nós.

como a contenção para o controle de acesso ao meio da camada de enlace e o cálculo da melhor rota de acordo com novos parâmetros da camada física.

\section{AGRADECIMENTOS}

Os autores agradecem o apoio financeiro da Agência Nacional do Petróleo - ANP - e da Finaciadora de Estudos e Projetos - FINEP - por meio do Programa de Recursos Humanos para o Setor Petróleo, Gás Natural e Biocombustíveis - PRH-ANP/MCT - (PRH10/UTFPR).

\section{REFERÊNCIAS}

[1] Q. Zhang and Y-Q. Zhang, "Cross-Layer Design for QoS Support in Multihop Wireless Networks," Proceedings of the IEEE, vol. 96, no. 1, pp. 64-76, Jan. 2008 .

[2] I.F. Akyildiz and X. Wang, "Cross-Layer Design in Wireless Mesh Networks", IEEE Transactions on Vehicular Technology, vol. 57, no. 9, pp. 1061-1076, Mar. 2008.

[3] X. Lin, N.B. Shroff, and R. Srikant, "A tutorial on cross-layer optimization in wireless networks," IEEE Journal on Selected Areas in Communications, vol. 24, n.8, pp. 1452-1463, Aug 2006.

[4] L. Iannone, R. Khalili, K. Salamatian, and S. Fdida, "Cross-layer routing in wireless mesh networks," 1st International Symposium on Wireless Communication Systems, pp. 319-323, 2004.

[5] D.E. Barreto and S.S. Chiu, "Decomposition Methods for Cross-Layer Optimization in Wireless Networks," Proc. of The IEEE Wireless Communications and Networking Conference (WCNC), pp. 270-275, 2007.

[6] John Boyer, David D. Falconer, and Halim Yanikomeroglu, "Multihop diversity in wireless relaying channels", IEEE Transactions on Communications, vol. 52, no. 10, pp. 1820-1830, October 2004.

[7] J. S. Thompson, P. M. Grant, and Y. Fan, "An introduction to multi-hop multi-antenna communications," Frequenz (Journal of RF-Engineering and Telecommunications), vol. 60, no. 5-6, pp. 103-106, 2006.

[8] D. Soldani and S. Dixit, "Wireless relays for broadband access", IEEE Communications Magazine, vol. 46, no. 3, pp 58-66, Mar 2008.

[9] S. Basagni et al., Mobile Ad Hoc Networking, IEEE Press and John Wiley Sons, 2003

[10] R. Bruno, M. Conti, and E. Gregori, "Mesh networks: commodity multihop ad hoc networks," IEEE Communications Magazine, vol. 43, no. 3, pp. 123-131, Mar. 2005.

[11] I. F. Akyildiz and X. Wang, "A survey on wireless mesh networks," IEEE Communications Magazine, vol. 43, no. 9, pp. S23-S30, Sep. 2005.

[12] T. H. Cormen, C. E. Leiserson, R. L. Rivest, and C. Stein, Introduction to Algorithms, Second Edition. MIT Press and McGraw-Hill, 2001. ISBN 0-262-03293-7. Section 24.1: The Bellman-Ford algorithm, pp.588-592.

[13] E. W. Dijkstra, A note on two problems in connexion with graphs, Numerische Mathematik, Dec 1959.
[14] Z. Wang and J. Crowcroft, "Quality-of-Service Routing for Supporting Multimedia Applications," IEEE Journal on Selected Areas in Communications, vol. 14, no. 7, pp. 1288-1234, Sep 1996.

[15] I. Chlamtac, M. Conti, and J.J.-N. Liu, "Mobile ad hoc networking: imperatives and challenges," Ad hoc Networks, Elsevier, vol. 1, 2003.

[16] I.F. Akyildiz, X.Wang, and W.Wang, "Wireless mesh networks: a survey," Computer Networks, vol.47, issue 4, Mar 2005.

[17] S. Hares, H. Yanikomeroglu, and B. Hashem, "Multi-hop relaying with diversity in peer-to-peer networks," Word Wireless Research Forum (WWRF) Meeting, n.9, Zurich, July 2003.

[18] S. Haykin and M. Moher, Modern Wireless Communications, Pearson Education, Upper Saddle River, NJ, 2005.

[19] D. Tse and P. Viswanath, Fundamentals of Wireless Communication, Cambridge University Press, 2005.

[20] S. M. Alamouti, "A simple transmit diversity techniques for wireless communications," IEEE Journal on Selected Areas in Communications, vol. 16, no. 8, pp. 1451-1458, Oct. 1998.

[21] V. Tarokh, H. Jafarkhani, and A. Calderbank, "Space-time block codes from orthogonal designs," IEEE Transactions on Information Theory, vol. 45, no. 05, pp. 1456-1467, July 1999.

[22] G. J. Foschini, "Layered space-time architecture for wireless communication in a fading environment when using multiple antennas," Bell Labs Technical Journal, vol. 1, no. 2, pp. 41-59, Autumn 1996.

[23] G. D. Golden, G. J. Foschini, R. A. Valenzuela, and P. W. Wolniansky, "Detection algorithm and initial laboratoy rsults using the V-Blast spacetime communication architecture," IEE Electronics Letters, vol. 35, no. 1, pp. 14-15, Jan. 1999.

[24] P. W. Wolniansky, G. J. Foschini, G. D. Golden, and R. A. Valenzuela, "V-BLAST: an architecture for realizing very high data rates over the rich-scattering wireless channel," Proc. Int. Symp. on Signals, Systems, and Electronics , pp. 295-300, Sept 1998.

[25] S. B. Wicker, Error Control Systems for Digital Communication and Storage, Englewood Cliffs: Prentice Hall, 1995. 